\title{
OBEDIENCIA AL DERECHO Y LEGITIMIDAD ESTATAL: UN ANÁLISIS DE LA TEORÍA DEL DEBER NATURAL DE OBEDECER INSTITUCIONES JUSTAS SEGÚN JEREMY WALDRON
}

\author{
OBEDIÊNCIA AO DIREITO E LEGITIMIDADE ESTATAL: UMA ANÁLISE DA \\ TEORIA DO DEVER NATURAL DE OBEDECER INSTITUIÇÕES JUSTAS \\ SEGUNDO JEREMY WALDRON
}

\section{OBEDIENCE TO LAW AND STATE LEGITIMACY: AN ANALISIS OF THE THEORY OF NATUAL DUTY OF OBEYING JUST INSTITUTIONS ACCORDING TO JEREMY WALDRON}

\begin{abstract}
CÉsAR BAENA
Abogado y Doctorando en Derecho y Ciencias Sociales (U.N.C.). Asesor en la Comisión de Asuntos Constitucionales de la Legislatura de la Provincia de Córdoba, Argentina. Profesor de Derecho Administrativo y Derecho Procesal Público (US21). Miembro del programa de investigación Autonomía, Control Social y Derechos Fundamentales (SECYT-U.N.C. y

US21). Córdoba, Argentina. baenaestudio@gmail.com
\end{abstract}

\begin{abstract}
RESUMEN
El presente trabajo analiza la plausibilidad de la teoría del deber natural de obedecer instituciones justas, como una justificación de la obligación de obedecer el derecho, según Jeremy Waldron. Para ello, el trabajo señala que dicha teoría está basada en un enfoque procedimental de la legitimidad, i. e., uno según el cual el procedimiento democrático tiene un valor intrínseco derivado del respeto a la igualdad política y al igual derecho a la participación. A partir de los argumentos que se dirigen a demostrar el deber natural de obedecer las instituciones que resuelven nuestros conflictos, en el marco de lo que Waldron llama las "circunstancias de la política", podrá apreciarse que es posible justificar no sólo la legitimidad de las decisiones coercitivas de un Estado democrático, sino también la obediencia política al derecho.
\end{abstract}

Palabras-clave: Deber natural. Legitimidad, Obediencia. Procedimiento democrático.

\section{RESUMO}

O presente trabalho analisa a plausibilidade da teoria do dever natural de obedecer instituições Justas, como justificação da obrigação de obedecer ao direito, Segundo Jeremy Waldren. Para isso, o trabalho esclarece que ttal teoria está baseada em um enfoque procedimental da legimidade, Segundo a qual o procedimento democrático possu um valor intrínseco derivado do respeito à igualdade política e ao direito à participação. A partir dos argumentos que demonstram o dever natural de obedecer às instituições que resolvem nossos conflitos, conforme com o que Waldron denomina de "circunstâncias da política", poderá ser apreciado que é possível justificar não apenas a legitimidade das decisões coercitivas de um Estado democrático, mas também a obediência política ao direito.

Palavras-chave: Dever Natural. Legitimidade. Obediência. Procedimento democratico.

\begin{abstract}
This paper analyzes the plausibility of the theory of the natural duty of obeying just institutions, as a justification of the obligation to obey the law, according to Jeremy Waldron. So, the work points that this theory is based on an approach of procedural legitimacy, i.e. one according to which the democratic procedure has an intrinsic value derived from respect for political equality and the equal right to participation. Based on the arguments that are
\end{abstract}




\section{政

OBEDIENCIA AL DERECHO Y LEGITIMIDAD ESTATAL: UN ANÁLISIS DE LA TEORÍA DEL DEBER NATURAL DE OBEDECER INSTITUCIONES JUSTAS SEGÚN JEREMY WALDRON

aimed at demonstrating the natural duty to obey the institutions that resolve our conflicts, within the framework of what Waldron calls the "circumstances of politics", it can be shown that it is possible to justify not only the legitimacy of the decisions of a democratic state, but also political obedience to the law.

Keywords: Natural duty. Legitimacy. Obedience. Democratic Procedure.

\section{SUMARIO}

INTRODUCCIÓN; 1 DESAFÍOS DE LA TEORÍA DEL DEBER NATURAL DE OBEDECER INSTITUCIONES JUSTAS; 1.1 Una alternativa al voluntarismo y al "juego limpio"; 1.2 Obligación política y legitimidad del Estado; 2 LA TEORÍA DEL DEBER NATURAL A PARTIR DE RAWLS; 3 DEBER NATURAL Y CIRCUNSTANCIAS DE LA POLÍTICA; 4 JUSTICIA EN EL PROCEDIMIENTO; CONCLUSIÓN; REFERENCIAS.

\section{INTRODUCCION}

El presente trabajo plantea un análisis acerca de la teoría del deber natural de obedecer instituciones justas (deber natural, de aquí en adelante), sostenida por autores como John Rawls y Jeremy Waldron, y entendida como una alternativa para justificar la obediencia al derecho. Particularmente intentaré una defensa de la versión procedimentalista de Waldron. En su formulación general, esta teoría también presupone una tesis acerca de la relación entre razones para justificar la legitimidad del Estado y las razones para la obediencia política. Conforme a ella, un ciudadano tiene el deber de obedecer solamente las normas de un Estado justo. La pregunta que aparece entonces es ¿qué debe entenderse por un “Estado justo”?

Al respecto, la concepción de Rawls sostenida en su Teoría de la Justicia ${ }^{1}$ responde que, en el marco de la justicia como imparcialidad, el Estado no sólo debe ocuparse de un esquema de libertades sustantivas, sino también de lo que se denomina "justicia política". Este aspecto de la justicia requiere de una constitución que satisfaga los requerimientos de igual libertad en el derecho de los ciudadanos a tomar parte y a determinar el resultado del proceso constitucional que establece las leyes que ellos han de obedecer. ${ }^{2}$

El fundamento de un procedimiento participativo e igualitario, según Rawls, no sería entonces sólo de índole técnica -esto es, acercarnos de la mejor forma posible al resultado sustantivo correcto- sino también de índole moral -dado por el valor intrínseco de la participación, de modo que se reduce el rango de procedimientos políticos aceptables-. No

\footnotetext{
${ }^{1}$ RAWLS, John. Teoría de la justicia. México: Fondo de Cultura Económica, 2006.

${ }^{2}$ Ibídem. p. 210.
} 


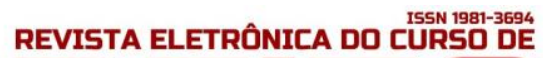

OBEDIENCIA AL DERECHO Y LEGITIMIDAD ESTATAL: UN ANÁLISIS DE LA TEORÍA DEL DEBER NATURAL DE OBEDECER INSTITUCIONES JUSTAS SEGÚN JEREMY WALDRON

obstante, el procedimiento no tiene un lugar prioritário en el esquema de libertades básicas. Esto queda más claro en su obra Liberalismo Político, en donde directamente concede a las libertades políticas un rol meramente instrumental. ${ }^{3}$

Pero el enfoque de Rawls no es el único que puede brindarse acerca de la idea de un “Estado justo”. Waldron, precisamente, defiende una idea de legitimidad de la decisión política basada en el procedimiento democrático mayoritario. Esto parece un avance teórico, al menos si se considera la preocupación de algunos autores por los enfoques que en general sólo brindan justificaciones instrumentales a la cuestión de la obediencia a las reglas. ${ }^{4}$

En la teoría de Waldron, en efecto, el valor intrínseco de la decisión democrática sí tiene una cierta prioridad con respecto a los resultados correctos -i. e., en los términos de un resultado sustantivo-. De allí que el autor resulta ser un acérrimo crítico del control judicial de las leyes y del atrincheramiento de derechos, precisamente porque considera que ambos mecanismos, al ser de carácter contramayoritario, no respetan el derecho a la igualdad política. ${ }^{5}$

En lo que sigue intentaré desarrollar de qué manera resulta plausible una teoría del deber natural como la de Waldron, basada en un enfoque de la legitimidad procedimental, i. e. uno según el cual el procedimiento democrático tiene un valor intrínseco derivado del respeto a la igualdad política y al igual derecho a la participación. A partir de los argumentos que se dirigen a demostrar el deber natural de obedecer las instituciones que resuelven nuestros conflictos, en el marco de lo que Waldron llama las "circunstancias de la política", podrá apreciarse que es posible justificar, no sólo la legitimidad de las decisiones coercitivas de un Estado democrático, sino también la propia obediencia política al derecho.

En el apartado siguiente (1), se analizará la importancia de la teoría del deber natural en su versión general, como superadora de otras teorías en el plano de la justificación de la obligación política (1.1) y como una opción para abordar la relación entre la obligación de obedecer el derecho y la legitimidad estatal (1.2). En al apartado 2), se señalarán las objeciones efectuadas a la teoría del deber natural originaria y se presentará parte del abordaje que efectúa Waldron al respecto. Luego (apartado 3), me detendré en las conexiones entre la idea

\footnotetext{
${ }^{3}$ RAWLS, John. Liberalismo político. México: Fondo de Cultura Económica, 2006. p. 278.

${ }^{4}$ Véase, por ejemplo, la crítica de Shapiro a la teoría de Raz sobre la autoridad como servicio, por considerarla una concepción puramente instrumental que soslaya el valor intrínseco de la toma de decisiones democrática. SHAPIRO, Scott J. Autoridad. Revista Derecho del Estado, Universidad Externado de Colombia, No. 31, julio-diciembre de 2013. p. 69.

${ }^{5}$ WALDRON, Jeremy. Derecho y desacuerdos. Madrid-Barcelona: Marcial Pons, 2005. pp. 305 y ss.
} 


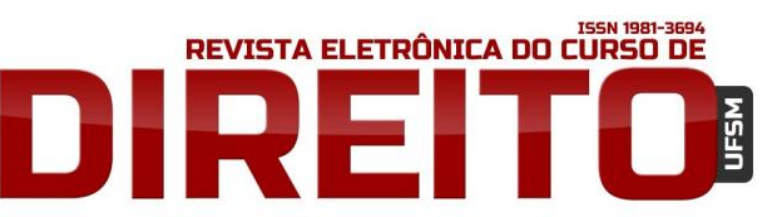

OBEDIENCIA AL DERECHO Y LEGITIMIDAD ESTATAL: UN ANÁLISIS DE LA TEORÍA DEL DEBER NATURAL DE OBEDECER INSTITUCIONES JUSTAS SEGÚN JEREMY WALDRON

de circunstancias de la política y la justificación del deber natural de obediencia. Finalmente, en el apartado 4) analizaré las implicancias de la legitimidad entendida desde el punto de vista procedimental, i. e., el del valor intrínseco del voto mayoritario.

\section{DESAFÍOS DE LA TEORÍA DEL DEBER NATURAL}

\subsection{Una alternativa al voluntarismo y al "juego limpio"}

La teoría del deber natural ha asumido al menos dos desafíos puntuales. En primer lugar, y ya que pretende ser una teoría justificadora del deber de obediencia al estado, se ha enfrentado particularmente a dos tipos de teorías: las voluntaristas y la del juego limpio.

Las teorías voluntaristas, en general, sostienen que las instituciones políticas tienen que estar justificadas en términos del consentimiento de los propios gobernados. De allí que están comúnmente asociadas a las teorías contractualistas (Hobbes, Locke, Rousseau), en el sentido de que justifican el gobierno en virtud de la realización, en el seno de la propia comunidad, de un pacto o autorización conferida a la autoridad estatal. Como sostiene Vilajosana, concepciones de este tipo parecen genuinamente respetuosas de la autonomía de las personas; no obstante, encuentran dificultades a la hora de demostrar la existencia real de algún tipo de acuerdo o consentimiento prestado. Todas las versiones del voluntarismo tienen problemas al respecto; desde las que sostienen que el consentimiento exigido debe ser expreso, hasta las teorías que, siguiendo a Locke, admiten el consentimiento tácito, e incluso las que sostienen que se trata de un acuerdo hipotético. Debe considerarse además, que la exigencia de consentimiento es alta, ya que requiere una voluntad unánime al momento de la asociación política, lo que en términos empíricos resulta imposible. Veamos cada una de estas versiones.

Conforme a la exigencia de un consentimiento expreso, resulta muy dificultoso determinar históricamente un momento en el cual pueda haber sido prestada la voluntad de todos los gobernados. También resulta muy difícil sostener que esa voluntad expresa pueda ser verificada luego. La teoría voluntarista que sostiene la necesidad de un consentimiento expreso queda descartada como plausible por esta razón: si el consentimiento es único y definitivo -como por ejemplo, si se piensa en la fundación constitucional- resulta rápidamente que él no ha sido prestado de manera unánime y que, además, suele ser muy remoto, con lo que se le opone rápidamente una objeción intergeneracional (v. gr. "el consentimiento es, en todo caso, 
de mis ancestros, pero no mío o de mis conciudadanos"). Tampoco puede buscarse un consentimiento expreso continuado en los casos de ejercicio de los derechos políticos -como el sufragio o la libertad de expresión-, ya que quedarían excluidos del acuerdo las personas que no toman parte en la toma de decisiones.

Señala Vilajosana que también el recurso al consentimiento tácito tiene inconvenientes. Alguien podría decir que una persona está obligada por el hecho de que ella elige, voluntariamente, residir en un determinado territorio afectado a la jurisdicción de una autoridad en concreto. Pero es extremadamente irrazonable exigir a una persona que abandone su lugar a los fines de que ello sirva como una forma de manifestación de su voluntad política. ${ }^{6}$ Apelar a la residencia es, entonces, muy poco plausible y no hay mayores recursos para defender esta forma de consentimiento. ${ }^{7}$

Por último, el consentimiento hipotético ha sido presentado como una alternativa, en el sentido de que plantea un marco racional abstracto a partir del cual resultaría que las personas, finalmente, elegirían vivir bajo las normas jurídicas de un estado. Lo que sucede es que allí no hay un acto de consentimiento como tal; y, lo que en cambio aparece, son consideraciones normativas que terminan confundiéndose con las mismas teorías de la justicia.

En segundo lugar, la teoría del deber natural busca ser una alternativa a la teoría del “juego limpio” sostenida por autores como Herbert L. A. Hart. Ésta última sostiene que el recibir beneficios de parte del Estado genera cargas frente a él, como la de obedecer las leyes que regularmente dicta, incluso independientemente del consentimiento. Esto presupone toda una concepción ontológica por parte de la teoría, como la de que todas las personas que viven bajo la autoridad de un Estado se benefician de ella. Existe un propósito común de supervivencia que la organización política, de esta manera, asegura a todos los gobernados. Este propósito, además, se encuentra amparado por un conjunto de "verdades obvias", que derivan en un "contenido mínimo de derecho natural". Por ejemplo, de la verdad obvia de que todas las personas, sin excepción, son vulnerables a los ataques físicos, se deriva la necesidad de adoptar normas que restrinjan el uso de la violencia; de la existencia limitada de recursos se infiere la necesidad de una regulación mínima del derecho de la propiedad; etc. ${ }^{8}$

\footnotetext{
${ }^{6}$ El recurso del cambio de residencia, o la reagrupación política -i. e., unirse con otros individuos para empezar otro gobierno- es propio de la teoría de Locke. Véase, LOCKE, John. Segundo Tratado sobre el Gobierno Civil. Madrid: Tecnos, 2006. pp. 114-115.

${ }^{7}$ VILAJOSANA, Josep M. Identificación y justificación del derecho. Madrid-Barcelona: Marcial Pons, 2007. pp. 130-131.

${ }^{8}$ HART, H. L. A. El concepto de derecho. Buenos Aires: Abeledo Perrot, 1998. pp. 239-247.
} 
La principal crítica del juego limpio proviene del lado del consentimiento. Así, Nozick sostiene que la imposición de cargas, como contrapartida de beneficios no requeridos por los gobernados, resulta ser injusta. ${ }^{9}$ De otro lado, si se mantiene la obligación moral de obediencia únicamente respecto de quienes aceptan recibir los beneficios, la teoría no sólo cede ante la idea de consentimiento, sino que también pierde toda pretensión de universalidad. ${ }^{10}$

En este marco, la teoría del deber natural se opone al voluntarismo y también a la teoría del juego limpio, las cuales son conocidas como teorías de la "obligación adquirida". Rawls sostiene que ni siquiera el consentimiento expreso ante instituciones claramente injustas originaría obligaciones. ${ }^{11}$ La justicia, desde este punto de vista, no puede admitir criterios que impliquen la realización de una contingencia, como haber prestado el consentimiento o haber recibido un beneficio. ${ }^{12}$ Pero además, como Waldron, sostiene que las mismas teorías de la obligación adquirida no son ajenas a la pretensión de justicia. ${ }^{13}$ Ellas son, de hecho, teorías normativas que alegan que es justo obedecer; o bien si se ha consentido con la conformación de la autoridad, o bien si se ha recibido algo de ella.

\subsection{Obligación política y legitimidad del Estado}

La teoría del deber natural también aborda la cuestión entre la obediencia al derecho y la legitimidad del Estado, aspecto que resulta fácil de advertir en la teoría de Waldron. La pregunta acerca de las razones para obedecer una norma dada, por lo general, se encuentra

\footnotetext{
9 Nozick proporciona varios ejemplos para demostrar lo implausible del "principio de equidad" según autores como Hart. Uno de ellos es expresado así: "Sería muy agradable tener conferencias de filosofía por la radio, las cuales podría uno sintonizar en cualquier momento, posiblemente a altas horas de la noche, cuando uno está cansado. Sin embargo, puede ser que no sea lo bastante agradable para que usted quiera ceder todo un día como conferenciante en el programa. Quiera usted lo que quiera ipueden los otros crearle una obligación de hacerlo, tomando la delantera y empezando el programa ellos mismos? En este caso usted puede escoger privarse del beneficio no encendiendo la radio; en otros casos los beneficios pueden ser inevitables." NOZICK, Robert. Anarquía, Estado y utopía. Buenos Aires: Fondo de Cultura Económica, 1991. p. 99.

${ }^{10} \mathrm{El}$ argumento de Nozick no me parece irrefutable. Es cierto que la mera idea de establecer cargas a cambio de beneficios es insuficiente; pero también resulta insatisfactorio requerir la mera voluntad del gobernado, no sólo desde el punto de vista normativo, sino también desde el punto de vista de la realidad práctica.

${ }^{11}$ RAWLS, John. Teoría de la justicia. p. 343.

12 Véase WALDRON, Jeremy. Special Ties and Natural Duties. En Philosophy and Public Affairs, Vol. 22, No. 1 , Winter, 1993. p. 3. Por cierto, es apropiado reconocer que hay un elemento de contingencia en la teoría del juego limpio, aun cuando sostiene que "todas las personas reciben beneficios del Estado".

13 Ídem. p. 4.
} 


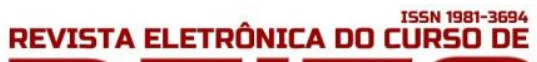

OBEDIENCIA AL DERECHO Y LEGITIMIDAD ESTATAL: UN ANÁLISIS DE LA TEORÍA DEL DEBER NATURAL DE OBEDECER INSTITUCIONES JUSTAS SEGÚN JEREMY WALDRON

asociada con la pregunta acerca de la legitimidad de quien ha dictado esa norma y exige luego su observancia. En el caso de las normas jurídicas, no sólo podemos preguntarnos acerca de qué razones justifican obedecer tales normas y en qué casos, sino también preguntarnos si su exigibilidad por el Estado resulta justa y con qué alcances.

Para la teoría del deber natural, es la legitimidad del Estado la que asume el lugar de requisito previo. Baste por ahora señalar que ella es en sí misma una condición de la obediencia. En términos de Waldron, resulta fundamental establecer si hay una buena razón para reconocer una organización política dada, como la única avocada a impartir justicia en un determinado territorio. ${ }^{14}$ Más adelante nos detendremos en los requisitos de legitimidad que señala este autor.

Lo cierto es que la relación entre ambas cuestiones -obediencia y legitimidad- ha sido asumida de otras maneras diferentes en la filosofía del derecho y en la filosofía política. Para algunos autores se trata del mismo problema, ya que consideran que el mismo hecho de que un Estado sea justo implica la obligación de obedecerlo; e, inversamente, si reconocemos que existe la obligación de obedecer las normas de un Estado, estamos con ello aceptando que dicho Estado es justo. Las razones son las mismas, y pueden ser de distinta índole. Como señala Vilajosana, una persona que adhiere a una tesis voluntarista acerca de la obediencia al derecho, deberá reconocer que todo Estado que respeta el consentimiento de los gobernados se encuentra moralmente justificado. ${ }^{15}$ Esta concepción debe ser endilgada a una gran cantidad de teóricos, precisamente en los casos en los que la distinción no ha sido advertida o no ha sido señalada categóricamente.

Otros autores, como Ronald Dworkin, consideran que primero es necesario - aunque no siempre suficiente- justificar la obediencia al derecho, para luego sostener que el Estado es legítimo. Dworkin, en este sentido, advierte acerca del requisito de la ley como fuente de "genuinas obligaciones". ${ }^{16}$ La idea de obligación genuina responde a una concepción asociativa que se halla basada en un rechazo al voluntarismo. ${ }^{17}$ Según este enfoque, las sociedades políticas en las que vivimos no son voluntarias, por lo tanto, tampoco los derechos que de ellas surgen pueden encontrar su origen en el consentimiento. En cambio, sobresale la reivindicación

\footnotetext{
${ }^{14}$ ídem. p. 22.

15 VILAJOSANA, op. cit. p. 127.

${ }^{16}$ DWORKIN, Ronald. El imperio de la justicia. Barcelona: Gedisa, 2012. p. 142.

${ }_{17}$ Cfr. IOSA, Juan F. La obligación de obedecer el derecho. En Enciclopedia de Filosofía y Teoría del Derecho. Instituto de Investigaciones Jurídicas de la Universidad Nacional Autónoma de México-UNAM, 2014. p. 1223.
} 
de una "experiencia moral compartida", inherente a todas las instituciones -desde luego, a las más informales como la familia, pero también a las de carácter político y jurídico- que remiten a un requisito de particularidad que, como veremos, el deber natural también aborda. Ahora bien, el asociativismo no considera lo suficiente el hecho de los desacuerdos al interior de las propias organizaciones. Soslayar los desacuerdos -más adelante veremos que, según Waldron, ellos forman parte de las "circunstancias de la política"- implica no sólo negarse a aceptar el voluntarismo, sino que también conlleva no respetar adecuadamente el lugar que cada miembro de la comunidad debe tener de manera igual en la toma de decisiones que resuelven nuestros conflictos. De allí que la idea de genuina obligación, como previa a la legitimidad no parezca satisfactoria.

\section{LA TEORÍA DEL DEBER NATURAL A PARTIR DE RAWLS}

Como hemos señalado, la teoría de Rawls es una teoría que separa conceptualmente el problema entre obediencia y legitimidad (aunque desde el punto de vista práctico son cuestiones dependientes); y que, además, ha sentado una importante posición respecto de la propia justificación del deber de obediencia. Esta teoría es la del deber natural. Ya que me interesa profundizar en esta teoría y revisar la versión de Waldron, empezaré por describirla brevemente señalando sus objeciones e incluyendo las respuestas puntuales que Waldron ofrece al respecto, ya que así se entenderá mejor su concepción procedimental en términos del deber natural.

Pues bien, según Rawls, tenemos un deber natural de obediencia al derecho, si sus normas se encuentran insertas en un régimen justo. En términos más precisos de Rawls, “desde el punto de vista de la justicia como imparcialidad un deber natural básico es el deber de justicia. Este deber nos exige apoyar y obedecer a las instituciones justas existentes que se nos aplican." 18

Hay dos objeciones a esta tesis de Rawls. La primera es conocida como la "exigencia de particularidad" u “objeción de la "especial lealtad”. El problema, según esta objeción, es que la tesis de Rawls no puede explicar el deber de una persona de obedecer el derecho de su propio estado en particular. Como señala Vilajosana, de todas las instituciones justas ipor qué tendríamos la obligación de apoyar y obedecer precisamente a instituciones políticas tales como

\footnotetext{
${ }^{18}$ RAWLS, Teoría de la justicia. p. 116.
} 


\section{(R)

OBEDIENCIA AL DERECHO Y LEGITIMIDAD ESTATAL: UN ANÁLISIS DE LA TEORÍA DEL DEBER NATURAL DE OBEDECER INSTITUCIONES JUSTAS SEGÚN JEREMY WALDRON

los estados? Y si debemos obedecer a los estados como tales, ¿por qué debemos una "lealtad” al nuestro? Y, suponiendo que tal lealtad es debida, ¿por qué honrarla necesariamente a través del cumplimiento de normas jurídicas? ${ }^{19}$

En este aspecto, Waldron demuestra que la objeción de la lealtad especial es débil si se la considera autónomamente, ya que la tesis de Rawls consiste en predicar el deber de obediencia respecto de las instituciones "que se nos aplican". El problema radica precisamente en la justificación acerca de que una determinada institución se nos aplique. Un movimiento insurgente podría exigirnos a cumplir con determinadas normas justas y ¿sólo por eso deberíamos obedecerle? Uno de los aportes más destacados en la reflexión de esta cuestión proviene de Simmons, para quien la idea de "aplicación" es ambigua y dilemática. Así, una institución que se nos aplique puede ser considerada en un sentido fuerte, es decir, a partir de la libre elección del individuo que la obedece. Por otro lado, la aplicación puede ser entendida en un sentido débil, es decir, a partir de una simple inclusión que del individuo hace la institución. La primera opción es problemática porque remite nuevamente a la idea de consentimiento; la segunda no lo es menos, ya que conforme a ella se debería admitir la imposición unilateral. ${ }^{20}$

Esta objeción es conocida como la “objeción de la aplicación”; y la respuesta de Waldron al respecto resulta fundamental a nuestros fines. La reconstrucción argumentativa que ofrece es, de hecho, la base de su concepción procedimentalista de la legitimidad de las instituciones políticas. Esta concepción, a su vez, se encuentra especialmente asociada con la teoría del deber natural, en tanto el desarrollo de ésta última le permitirá luego formular -a propósito de una analogía con la teoría rawlsiana- ${ }^{21}$ la idea de las "circunstancias de la política". Waldron se refiere con este concepto a la necesidad percibida por los miembros de una determinada comunidad de contar con un marco decisorio, o un curso de acción común, sobre las distintas cuestiones a las que en su seno surgen; a la par de los profundos desacuerdos de principio, muchas veces ineludibles e insalvables, entre los propios miembros.

\footnotetext{
${ }^{19}$ VILAJOSANA, op. cit. p. 164.

20 SIMMONS, A. John. Moral Principles and Political Obligations. Princeton: Princeton University Press, 1979. p. 147-148.

${ }^{21}$ Rawls acuña la idea de las circunstancias de la justicia, identificando como tales el altruismo limitado de los individuos y la escasez moderada de los recursos, que son las que hacen que la cooperación humana sea tanto posible, como necesaria. Véase RAWLS, Teoría de la justicia. pp. 1226-129.
} 


\section{DEBER NATURAL Y CIRCUNSTANCIAS DE LA POLÍTICA}

Pero la pregunta, después de todo, es si podemos avanzar de alguna manera ante el problema moral de que una institución determinada se nos aplique, con el solo hecho de alegar que nos encontramos ante una circunstancia de la política. Si la respuesta de la teoría del deber natural es satisfactoria en este sentido, las circunstancias de la política pueden justificar la obligación de obedecer una institución justa en la medida en que sus decisiones nos sean aplicables.

Según Waldron, está claro que un ciudadano $\mathrm{C}$, nacional del país $\mathrm{Y}$, puede estar obligado a obedecer las normas de un país $Z$, al menos cuando $Z$ es un país justo, así como sus normas. Supongamos que C viaja al país Z. Seguramente, ni bien arribe, deberá observar determinadas reglamentaciones aeroportuarias, o deberá pagar determinados impuestos al consumo de bienes y servicios, como el IVA. Ahora bien, ¿acaso las teorías del consentimiento o del juego limpio no son más satisfactorias al respecto? En todo caso, o bien se puede decir que $C$ consintió en ingresar al país $Z$, o bien se puede decir que es justo que él deba soportar determinadas cargas por los beneficios prestados por el país anfitrión. Waldron señala que, no obstante, no todos los casos son de este tipo. Podría pensarse que el ciudadano $C$, sin moverse de su país $\mathrm{Y}$, podría violar las normas del país Z (el ejemplo señalado por el autor es el de unos funcionarios franceses involucrados en un ataque contra un barco de la organización Greenpeace que ejercieron mecanismos extorsivos sobre la justicia neozelandesa para obtener determinados beneficios de impunidad). La obligación de obedecer o de apoyar el sistema de justicia de un país ajeno, como en el caso, no parece cubierta por las teorías voluntaristas o la del juego limpio.

Suponemos, a partir de aquí, que adherentes a las tres teorías comparten las mismas intuiciones sobre este último caso -básicamente, que hay una violación de las normas de un país ajeno-. Corresponde entonces a los proponentes del deber natural explicar por qué los hechos cometidos son incorrectos moralmente, independientemente de la voluntad de los autores.

Para Waldron, la explicación merece ser desglosada en tres niveles; los primeros dos de carácter abstracto y el tercero de carácter concreto. En el primer nivel, se advierte que existen principios de justicia que los propios individuos pueden sostener. $Y$ aquí ya aparece una primera oportunidad para el desacuerdo. Supongamos el principio $\mathrm{P}_{1}$; las mismas personas que lo 
reconocen como tal, pueden interpretarlo de diversa manera, con lo cual deberemos advertir que se trata de un principio de "rango limitado". El contractualismo kantiano resulta ser una explicación adecuada de cómo las personas se sirven de las instituciones y arreglos estatales para resolver sus conflictos. ${ }^{22}$ La cuestión moral en esto no es de carácter abierto, ya que los individuos deben aceptar todos aquellos arreglos dirigidos a evitar o disminuir los conflictos que surgen del uso propio de los recursos. ${ }^{23}$

En cuanto al segundo nivel, se debe señalar que todo principio de rango limitado, como $\mathrm{P}_{1}$, debe ser aplicado por alguien en particular que decida sobre el caso -la institución política L-, ya que -de lo contrario- el desacuerdo sobre el punto hace imposible una solución efectiva. Esta necesidad implica la existencia de otros principios $\left(P_{2}, P_{3}\right.$, etc.) como el que sostiene que el decisor debe actuar de manera imparcial, deliberada o razonable, etc.

\section{JUSTICIA EN EL PROCEDIMIENTO}

En el tercer nivel, se encuentra no sólo la concretización de los dos primeros niveles -la existencia de desacuerdos acerca de los principios y la necesidad de que alguien decida sobre el conflicto- sino también la cuestión clave para advertir el carácter procedimental que tiene la teoría del deber natural según Waldron. Como el autor señala,

[r]ápidamente nos enfrentamos con una comunidad de personas que pretenden hacer justicia en nuestro territorio. Para que puedan perseguir ese objetivo, ellas deben obtener un cierto grado de cumplimiento y apoyo de parte de nosotros. La teoría del derecho natural es que tienen derecho a ese cumplimiento y apoyo simplemente en virtud de la calidad de la organización que han reunido. ${ }^{24}$

Pero las circunstancias de la política, en este sentido, no agotan los requisitos de justificación de la obediencia. Según Waldron, hay dos condiciones adicionales al respecto. La primera es la condición de la efectividad. La segunda, como ya hemos señalado supra, es la condición de la legitimidad.

\footnotetext{
${ }^{22}$ En este sentido, sostiene Kant: "Del derecho privado en el estado natural resulta un postulado de Derecho público: 'Tú debes juntamente con los demás, en la relación de una coexistencia necesaria, salir del estado natural, para entrar en un estado de derecho, es decir, estado de una justicia distributiva.' La razón puede deducirse analíticamente de la noción del derecho en las relaciones exteriores por oposición [a] la violencia (violentia)." KANT, Immanuel. Principios metafísicos del derecho. Madrid: Librería de Victoriano Suárez, 1873. p. 161.

${ }^{23}$ Véase, WALDRON, Jeremy. Special Ties and Natural Duties. p. 14.

${ }^{24}$ Ídem, p. 20. La traducción me pertenece.
} 
En primer lugar, el nivel de concretizar la solución de los desacuerdos a partir de una determinada institucionalidad nos exige que la organización en cuestión sea realmente capaz de hacer justicia, es decir, efectiva. No resulta razonable esperar que los desacuerdos y las pretensiones de resolver los conflictos sean, al decir de Waldron, sólo parte de una "causa perdida". La propia comunidad, es decir el conjunto de individuos entre quienes el desacuerdo surge, tiene que estar de alguna manera preparada para aceptar la decisión que surgirá de la institución $L$ interviniente. Según Waldron, esto no implica una circularidad. No es lo mismo que decir que hay un deber de seguir principios tales como $P_{1}, P_{2}$ y $P_{3}$ sólo si la institución $L$ es efectiva y que, a la vez, la institución L es efectiva sólo si se siguen los principios referidos. La cuestión es que cada persona debe estar segura de que los demás están dispuestos a seguir los mismos principios, incluso antes de que cada uno piense que $L$ es efectiva y que de allí surge la obligatoriedad.

Por supuesto, la efectividad no resuelve por sí misma las cosas. En todo caso, sí llega a responder gran parte de la pregunta acerca de la justificación de que una institución dada se nos aplique. Lo que sucede, además, como ya hemos sugerido al mencionar el ejemplo de las normas dictadas por un grupo insurgente, es que puede haber más de una organización efectiva -institución o proto-institución L- que sea efectiva en estos términos.

Y aquí es donde debemos empezar a abordar la cuestión de la legitimidad como condición de la obediencia al derecho. Ante todo, debemos remarcar que una característica definitoria de la legitimidad de una institución, en las circunstancias de la política, es su exclusividad: no puede haber dos instituciones legítimas resolviendo el mismo asunto en el mismo territorio entre las mismas personas. Los argumentos de Waldron para justificar esta legitimidad exclusiva pueden resumirse como se sigue.

En primer lugar, es fundamental remarcar la importancia de las instituciones en su tarea de hacer justicia como tales. Siguiendo a Kant, las instituciones políticas contribuyen a la justicia en la medida que evitan o mitigan los desacuerdos y conflictos. ${ }^{25}$ Es cierto que para ser más rigurosos en la lectura de la teoría general de Waldron deberíamos decir que lo que se evita

\footnotetext{
${ }^{25}$ El principio básico de Kant según el cual "es menester ante todo entrar en un estado civil" es seguido por una explicación acerca de cómo la justicia en dicho estado se diferencia de la justicia en el estado natural. Sostiene el autor: "A la verdad, no por esto el estado natural debería ser un estado de injusticia (in justo, en el cual los hombres únicamente se tratasen según la medida exclusiva de sus fuerzas; pero es por lo menos un estado de justicia negativa (status justitie vacuus), en el cual, si el derecho fuese controvertido, no habría juez competente para dictar una legítima sentencia, en virtud de la cual cada uno pudiese obligar a otro a salir de ese estado de guerra y hacerle entrar en un estado jurídico." KANT, op. cit. pp. 165-166.
} 


\section{每

OBEDIENCIA AL DERECHO Y LEGITIMIDAD ESTATAL: UN ANÁLISIS DE LA TEORÍA DEL DEBER NATURAL DE OBEDECER INSTITUCIONES JUSTAS SEGÚN JEREMY WALDRON

Cesar BaEna

o mitiga es, en definitiva, el efecto de los desacuerdos y conflictos. Lo importante es reconocer que hay un deber moral respecto de la evitación de esos efectos. Los desacuerdos y el conflicto -sobre todo si se piensa en una hipótesis extrema de estado de naturaleza o de guerra como lo pensó Hobbes frente a la no existencia de autoridad $-{ }^{26}$ traen consigo el riesgo de la violencia, y con ella la muerte y el sufrimiento.

Segundo, la exclusividad termina por evitar un estado de guerra peor que el que podría existir ante la ausencia de una autoridad. Como sostiene Nozick, la pretensión de tener dos instituciones paralelas lleva a que las personas se organicen, con lo que la amenaza de violencia deja de ser la amenaza de un enfrentamiento de todos contra todos, para ser un enfrentamiento de alguna manera organizado que puede traer peores consecuencias.

Pero no sólo esto. Además del deber de evitación de la violencia organizada, es importante reconocer que la justicia es en gran parte un asunto de cooperación. Dicho de otro modo, la cooperación realiza la justicia en circunstancias en que ella es imposible de ser concebida individualmente. El hambre en una comunidad, por ejemplo, muy difícilmente podría acabarse de la mano de la caridad de unos pocos individuos. De hecho, podríamos dudar acerca de si, efectivamente y al margen de un resultado positivo logrado, se ha hecho justicia si sólo ha tenido lugar la preocupación aislada de algunas personas, sin que hubiera mediado colaboración u organización alguna.

En tercer lugar, resulta importante identificar las razones que hacen que debamos preferir una determinada forma de organización y ante lo cual debamos descartar otras opciones igualmente efectivas. Después de todo, el hecho de que requiramos efectividad no puede llevarnos a escoger la forma de institución más poderosa sin más; esto haría el criterio de legitimidad redundante. Según entiendo, aquí está lo novedoso del pensamiento de Waldron.

La teoría de la legitimidad de las instituciones políticas, debe aclararse, tiene en el autor en estudio un desarrollo bastante más amplio y, por igual motivo, bastante más autónomo de la teoría del deber natural. Nuevamente insistimos, como ya lo hiciera Vilajosana, esto no hace

\footnotetext{
${ }^{26}$ Recordemos la mirada de Hobbes sobre el punto: "Son tantos los peligros que amenazan a todos como consecuencia de la codicia y apetitos de cada hombre, que el que todos hayamos de protegernos y cuidar de nosotros está tan lejos de ser tomado en broma, que nadie puede ni quiere hacer otra cosa. Pues todo hombre está siempre deseoso de lograr lo que es bueno para él, y de rechazar lo que es malo; y quiere principalmente evitar el más grave de todos los males naturales, que es la muerte [...] Por lo tanto, el primer fundamento del derecho natural es éste: que todo hombre procure, en la medida de sus fuerzas, proteger su vida y sus miembros." HOBBES, Thomas. De Cive. Madrid: Alianza Editorial, 2000. p. 60. Un análisis sobre las semejanzas y diferencias entre Hobbes y Kant respecto de la idea de desacuerdos puede encontrarse en WALDRON, Jeremy. The Dignity of Legislation. New York: Cambridge University Press, 1999. pp. 42-46.
} 


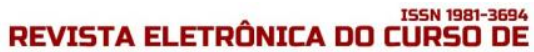

OBEDIENCIA AL DERECHO Y LEGITIMIDAD ESTATAL: UN ANÁLISIS DE LA TEORÍA DEL DEBER NATURAL DE OBEDECER INSTITUCIONES JUSTAS SEGÚN JEREMY WALDRON

que -incluso en el enfoque waldroniano- ambas sean cuestiones independientes. Al defender la teoría del deber natural Waldron se limita a responder que

parece ser que si la mayoría de las personas en un territorio están de acuerdo con que alguna organización $L$ es el sistema para mantener el orden e impartir justicia en ese territorio, entonces su consentimiento confiere legitimidad $L$ y nos proporciona una base para identificar a L como la institución que merece nuestro apoyo y lealtad. ${ }^{27}$

Sí hay un abordaje bastante clarificador al respecto, ya que Waldron reivindica que esto no implica una reintroducción del consentimiento en la justificación de la obediencia. No obstante, llega a afirmar que la cuestión pasa por una aceptación mayoritaria de la institución decisora y que, si bien ella está asociada a un uso determinado del consentimiento, no es en absoluto equivalente con la noción de consentimiento tal y como es empleada por las teorías voluntaristas. De hecho, el voluntarismo no es prima facie compatible con la idea de mayorías. ${ }^{28}$ En un sistema democrático, dadas las circunstancias de la política, resulta moralmente exigible observar las decisiones de una institución o autoridad determinada; resulta exigible incluso para aquellos que no han prestado un consentimiento -expreso o tácito- a su respecto, o no se han beneficiado concretamente por causa de la decisión. Pero esa exigibilidad procede sólo en los casos en los que se cumplen determinadas condiciones inherentes a una idea de justicia procedimental, observada en el marco de la toma de decisiones y constitutivas de la propia legitimidad democrática de la autoridad en cuestión.

En Derecho y desacuerdos, Waldron deja en claro en qué sentido considera que la decisión mayoritaria es el procedimiento más justo para tomar decisiones en el marco de las circunstancias de la política. Sostiene Waldron:

[r]espetar el hecho de los desacuerdos nos lleva a enfrentarnos a un problema de decisión en el que el individuo, a pesar de tener confianza en su propio punto de vista, no pasa a la acción partiendo del mismo [...] el principio de la decisión mayoritaria... afirma que respecto de cada individuo, el hecho de que dicho individuo respalde la opción $X$ es una opción para que el grupo adopte la opción $\mathrm{X}$, aun cuando existan desacuerdos. ${ }^{29}$

En cuanto a la importancia del procedimiento mayoritario para la igualdad política, Waldron remarca que el mayoritarismo implica el compromiso de dar igual peso a cada punto de

\footnotetext{
${ }^{27}$ WALDRON, Jeremy. Special Ties and Natural Duties. p. 25.

28 Ídem, pp. 25-26.

${ }^{29}$ WALDRON, Jeremy. Derecho y desacuerdos. pp. 136-137.
} 
vista; de hecho, el máximo peso compatible con un peso igual reconocido al resto de los individuos. Con esto, Waldron se acerca bastante al principio de iguales libertades rawlsiano, ${ }^{30}$ aunque lo hace en una estricta aplicación sobre el procedimiento, reconociendo que es posible que la decisión mayoritaria no sea infalible. Incluso, aun cuando ella sea susceptible de producir resultados incorrectos en términos de justicia sustantiva, el procedimiento democrático igualitario resulta preferible. Esto, no sólo porque respeta igualitariamente a cada individuo que ha participado en la toma de decisión, sino porque la propia circunstancia de los desacuerdos hará que encontremos nuestras concepciones divididas acerca de la justicia sustantiva que ha surgido del proceso político autoritativo.

\section{CONCLUSIÓN}

El trabajo ha pretendido desarrollar una defensa de la teoría del deber natural, la que fuera sostenida primero por Rawls y luego por Waldron, como mejor justificación de la obediencia al derecho. También ha sostenido la necesidad de distinguir que en el seno de la propia teoría hay una divergencia importante, pues los criterios para definir la justicia que funciona como precondición de la obediencia son muy distintos en Rawls con respecto a Waldron. De esta manera, se ha tomado como punto de referencia la teoría waldroniana, ya que defiende una concepción procedimental de justicia, defensora del voto mayoritario, el cual se encontraría moralmente justificado en el marco de las circunstancias de la política explicadas.

No ha sido el objeto principal de este trabajo, pero resulta importante dejar el debate abierto a otros argumentos que pretenden considerar el valor moral de las instituciones cuando, sin estar determinadas por el voto mayoritario o democrático, se presentan como respetuosas de la igualdad política. La propia teoría de Rawls, a partir de la defensa de determinadas estructuras como apropiadas para la realización de la razón pública interpela al respecto de forma significativa. De hecho, una buena parte de las teorías deliberativas se oponen al procedimentalismo de Waldron, entre otras razones por considerar que su descripción de los desacuerdos va demasiado lejos y es, en parte, radical.

No obstante, los méritos de la teoría waldroniana son dignos de reconocimiento, ya que logra un abordaje muy sólido de las circunstancias de la política en su vinculación con el deber

\footnotetext{
${ }^{30}$ Rawls no descarta el carácter igualitario de la decisión mayoritaria, pero ella ocupa un lugar secundario incluso como mecanismo procesal. Véase, RAWLS. Teoría de la justicia. p. 325.
} 


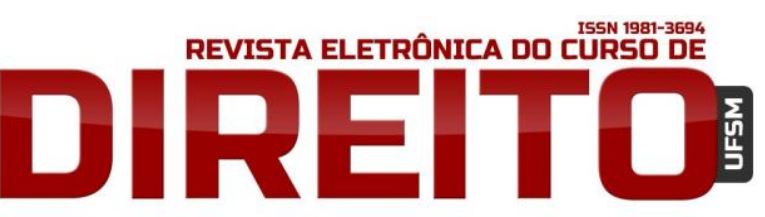

OBEDIENCIA AL DERECHO Y LEGITIMIDAD ESTATAL: UN ANÁLISIS DE LA TEORÍA DEL DEBER NATURAL DE OBEDECER INSTITUCIONES JUSTAS SEGÚN JEREMY WALDRON

natural de obedecer las decisiones colectivas cuando ellas reúnen determinadas condiciones de legitimidad. No es una cuestión menor que, en cuanto al análisis de dicha legitimidad, Waldron tenga presente el respeto igualitario de las preferencias políticas de los individuos; i. e., la defensa del voto y de la igualdad política entendidos en términos liberales. Este mérito se muestra aún mejor cuando se recuerda el desafío permanente que plantea la tensión entre la teoría liberal y la defensa de la democracia mayoritaria.

\section{REFERÊNCIAS}

DWORKIN, Ronald. El imperio de la justicia. Barcelona: Gedisa, 2012.

HART, H. L. A. El concepto de derecho. Buenos Aires: Abeledo Perrot, 1998.

HOBBES, Thomas. De Cive. Madrid: Alianza Editorial, 2000.

IOSA, Juan F. La obligación de obedecer el derecho. En Enciclopedia de Filosofía y Teoría del Derecho. Instituto de Investigaciones Jurídicas de la Universidad Nacional Autónoma de México UNAM, 2014. pp. 1193-1245.

KANT, Immanuel. Principios metafísicos del derecho. Madrid: Librería de Victoriano Suárez, 1873.

LOCKE, John. Segundo Tratado sobre el Gobierno Civil. Madrid: Tecnos, 2006.

NOZICK, Robert. Anarquía, Estado y utopía. Buenos Aires: Fondo de Cultura Económica, 1991.

RAWLS, John. Teoría de la justicia. México: Fondo de Cultura Económica, 2006.

RAWLS, John. Liberalismo político. México: Fondo de Cultura Económica, 2006.

SHAPIRO, Scott J. Autoridad. Revista Derecho del Estado, Universidad Externado de Colombia, No. 31, julio-diciembre de 2013. pp. 5-77.

SIMMONS, A. John. Moral Principles and Political Obligations. Princeton: Princeton University Press, 1979.

VILAJOSANA, Josep M. Identificación y justificación del derecho. Madrid-Barcelona: Marcial Pons, 2007.

WALDRON, Jeremy. Special Ties and Natural Duties. En Philosophy and Public Affairs, Vol. 22, No. 1, Winter, 1993, pp. 3-30. 
OBEDIENCIA AL DERECHO Y LEGITIMIDAD ESTATAL: UN ANÁLISIS DE LA TEORÍA DEL DEBER NATURAL DE OBEDECER INSTITUCIONES JUSTAS SEGÚN JEREMY WALDRON

Cesar BAEnA

WALDRON, Jeremy. The Dignity of Legislation. New York: Cambridge University Press, 1999.

WALDRON, Jeremy. Derecho y desacuerdos. Madrid-Barcelona: Marcial Pons, 2005.

Artigo Convidado.

\section{COMO CITAR O ARTIGO (ABNT)}

BAENA, César. OBEDIENCIA AL DERECHO Y LEGITIMIDAD ESTATAL: UN ANÁLISIS DE LA TEORÍA DEL DEBER NATURAL DE OBEDECER INSTITUCIONES JUSTAS SEGÚN JEREMY WALDRON. Revista Eletrônica do Curso de Direito da UFSM, Santa Maria, RS, v. 12, n. 2, p. 675-691, ago. 2017. ISSN 1981-3694. Disponível em: <https://periodicos.ufsm.br/revistadireito/article/view/28341>. Acesso em: dia mês. ano. doi:http://dx.doi.org/10.5902/1981369428341. 